\title{
A Decisive Filtering Selection Approach For Improved Performance Active Noise Cancellation System
}

\author{
Abha Nyati ${ }^{1}, \mathrm{~S}$ K Dargar ${ }^{2}, * *$ Deepak Vyas \\ ${ }^{I}$ (Department of E \& C Engineering, Pacific University, (PAHER) Udaipur, Rajasthan, India) \\ ${ }^{2}$ (Faculty, Department of E \& C Engineering, SPSU University, Udaipur, Rajasthan, India) \\ ** (Faculty, Department of E \& C Engineering, Pacific Institute of Technology, Udaipur, Rajasthan, India)
}

\begin{abstract}
In this work we present a filtering selection approach for efficient ANC system. Active noise cancellation (ANC) has wide application in next generation human machine interaction to automobile Heating Ventilating and Air Conditioning (HVAC) devices. We compare conventional adaptive filters algorithms LMS, NLMS, VSLMS, VSNLMS, VSLSMS for a predefined input sound file, where various algorithms run and result in standard output and better performance. The wiener filter based on least means squared (LMS) algorithm family is most sought after solution of ANC. This family includes LMS, NLMS, VSLMS, VSNLMS, VFXLMS, FXsLMS and many more. Some of these are nonlinear algorithm, which provides better solution for nonlinear noisy environment. The components of the ANC systems like microphones and loudspeaker exhibit nonlinearities themselves. The nonlinear transfer function create worse situation. This is a task which is some sort of a prediction of suitable solution to the problems. The Radial Basis Function of Neural Networks (RBF $N N)$ has been known to be suitable for nonlinear function approximation [1]. The classical approach to RBF implementation is to fix the number of hidden neurons based on some property of the input data, and estimate the weights connecting the hidden and output neurons using linear least square method. So an efficient novel decisive approach for better performing ANC algorithms has been proposed.
\end{abstract}

Keywords - Adaptive filters, Winner filter ANC, Least mean square, N LMS, VSNLMS, RBF.

\section{OVERVIEW}

Acoustic Noise Cancellation is a method for reducing undesired noise. It is achieved by introducing a canceling "anti-noise" wave through secondary sources. These secondary sources are interconnected through an electronic system using a specific signal processing algorithm for the particular cancellation scheme. Noise cancellation makes use of the notion of destructive interference. When two sinusoidal waves are superimposed, the resultant waveform depends on the frequency amplitude and relative phase of the two waves. If the original wave and the inverse of the original wave encounter at a junction at the same time, total cancellation occurs. The challenges are to identify the original signal and generate the inverse noise cancellation on inverse wave without delay in all directions where noises interact and superimpose. We meet in our everyday life. Echo phenomena are interesting and entertaining, but their presences in communication networks are undesirable and represent a serious problem. Echo is delayed and degraded version of original signal which travels back to its source after several reflections or because of some other reason. Nature of echo signal can be either acoustic or electrical, and in order to reduce its undesired effect we employ echo cancellers. Design of echo cancellers requires an application of adaptive filter theory. Echo cancellers must work within specific time limits so adaptive algorithms must provide fast convergence of filter parameters. We've been applying echo cancellers successfully for many years, but we always tend to improve them and increase their efficiency.

The wiener filter based least means squared (LMS) algorithm family is most sought after solution of ANC. This family includes LMS , Fx-LMS, VFx-LMS, FsLMS and many more . Then there are Kalman filter algorithms which are basically based on recursive least square algorithm. Some of these are nonlinear algorithm, which provides better solution for non linear noisy environment. The components of the ANC systems like microphones and loudspeaker exhibit nonlinearities themselves. The non linear transfer function of primary and secondary path may itself create worse situation.

\section{BACKGROUND LITERATURE REVIEW}

Active Noise Control (ANC) includes an electromechanical system or electro acoustic system that cancels the unwanted noise based on the principle of superposition of an anti-noise wave of equal amplitude. The customary ways for active noise cancellers uses passive techniques such as enclosures, barriers, and silencers to attenuate the undesired noise. These passive silencers are valued for their high attenuation over a broad frequency range. However, they are relatively large, costly, and ineffective at low frequencies. Mechanical vibration is another related type of noise that commonly creates problems in all areas of transportation and manufacturing, as well as with many household appliances. S.M. Kuo and D. R. Morgan 
(1996) in their paper have emphasized the practical aspects of ANC systems in terms of adaptive algorithms and DSP implementations for real-world applications [4]. Widrow and Hoff (1960) developed the least mean square algorithm (LMS). This algorithms is a member of stochastic gradient algorithm algorithms, and because of robustness and low computational complexity, it has been used in wide spectrum of applications [6]. Least square solution is not very practical in the actual implementation of adaptive filters, this is because we know all the past samples of the input signal, and as well the desired output signal must be available at every iteration. Performance with less computational complexity compared to the Second-order VFXLMS algorithm [9]. Debi Prasad das and G. panda in 2004 has shown a new approach for nonlinear processes using Filtered-s LMS (FsLMS). They proved that using a nonlinear controller for a linear device can achieve superior performance when the secondary path is linear non minimum phase and the reference noise is non-gaussian and predictable.

\section{PROCEDURE AND APPROACH}

The experimental work has been done on MATLAB, audio processor, capable to perform real time processing on signals. Various program based on individual algorithm were written first e.g. LMS, NLMS, FSLMS, VSLMS. Each of them is considered as a separate hidden neuron of network. In case two primary noise signal is chosen to be a logistic chaotic type $x(n+1)=\lambda x(n)[1-x(n)] \lambda=4, x(0)=0,9[2]$

All the neurons were allowed to run for 600,1200 and 1800 iterations, and respective obtained results were compared with standard threshold value. LMS algorithm weight update equation is as

$$
w(n+1)=w(n)-\mu e(n) v(n)
$$

$\mathrm{v}(\mathrm{n})=\mathrm{x}(\mathrm{n})^{*} \mathrm{a}(\mathrm{n}) \quad$ where $\mathrm{a}(\mathrm{n})$ is approximation of secondary path transfer function. $\mathrm{w}(\mathrm{n})$ if filter weight,$\mu$ is step size factor[2]. The calculation of threshold depends upon number of parameter and it may vary according to surrounding situation and instantaneous noise characteristics. We had adopted threshold parameter $\delta \mathrm{k}$ Which was based on following equations. For each input $\mathrm{Xn}$

$$
\delta_{k}(X n)=\exp \left(-1 / \sigma 2\left(\left\|X_{N^{-}} \mu_{K}\right\|^{2}\right)\right)
$$

Where $\sigma$ is variance, $\mu$ is is weight factor of particular neuron [5]. This approach is based on technique of RBF neural network. We are using this technique to prune less effective neurons from network and finally running systems with successful neuron only. In different conditions different neuron may give better results. The survival chances of neurons depend upon its algorithm efficiency in given circumstances. The step $\mu$ was size varied factor between $.05,0.125$ and 0.5 depending upon the optimization required between complexity and amount of noise cancellation. We have done experimental studies on various inputs for different algorithms e.g. LMS, NLMS, VSLMS, VSNLMS etc. Finally for simulations trial we opted one particular acoustic signal of linear nature, which was producing a sound in the frequency range of low decibels.

IV.
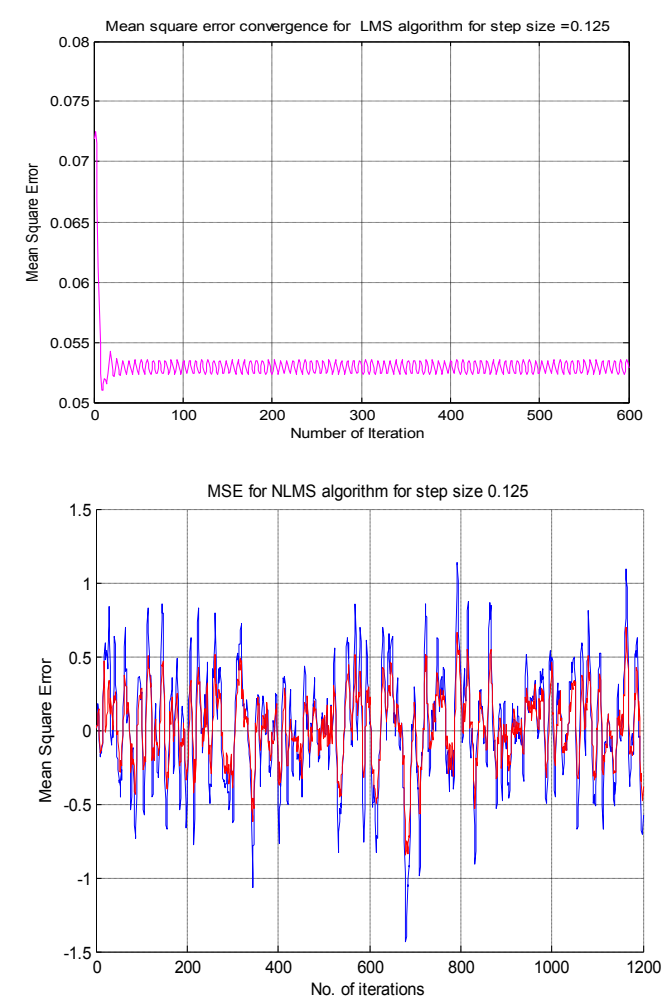

\section{RESULT AND ANALYSIS}
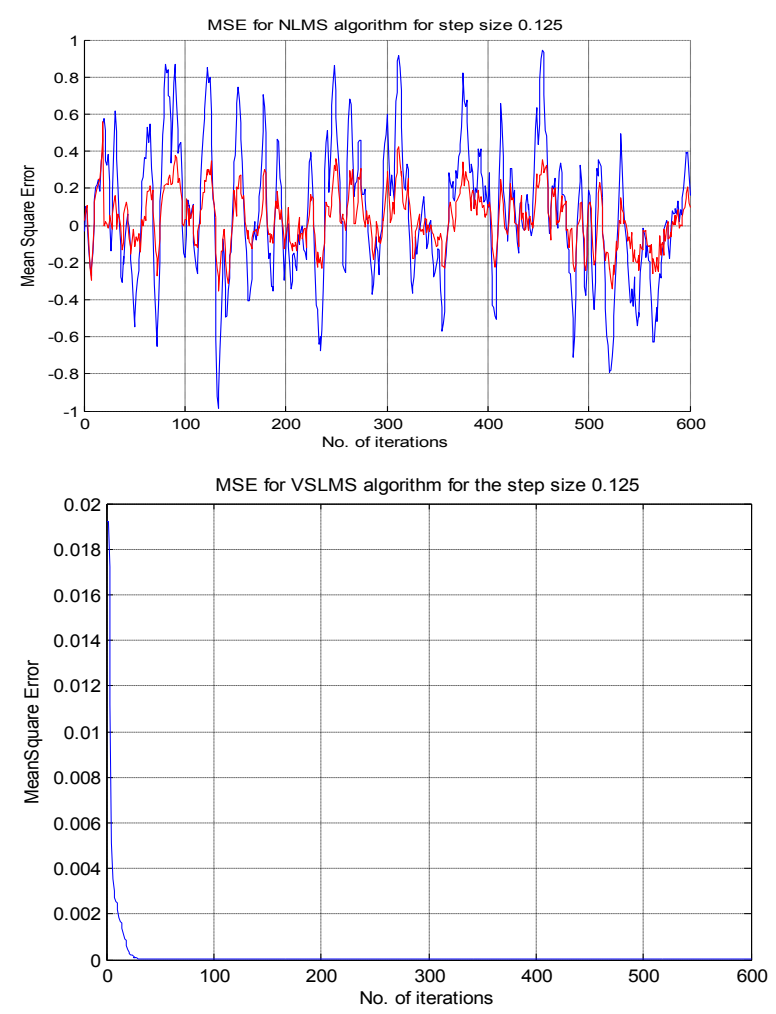

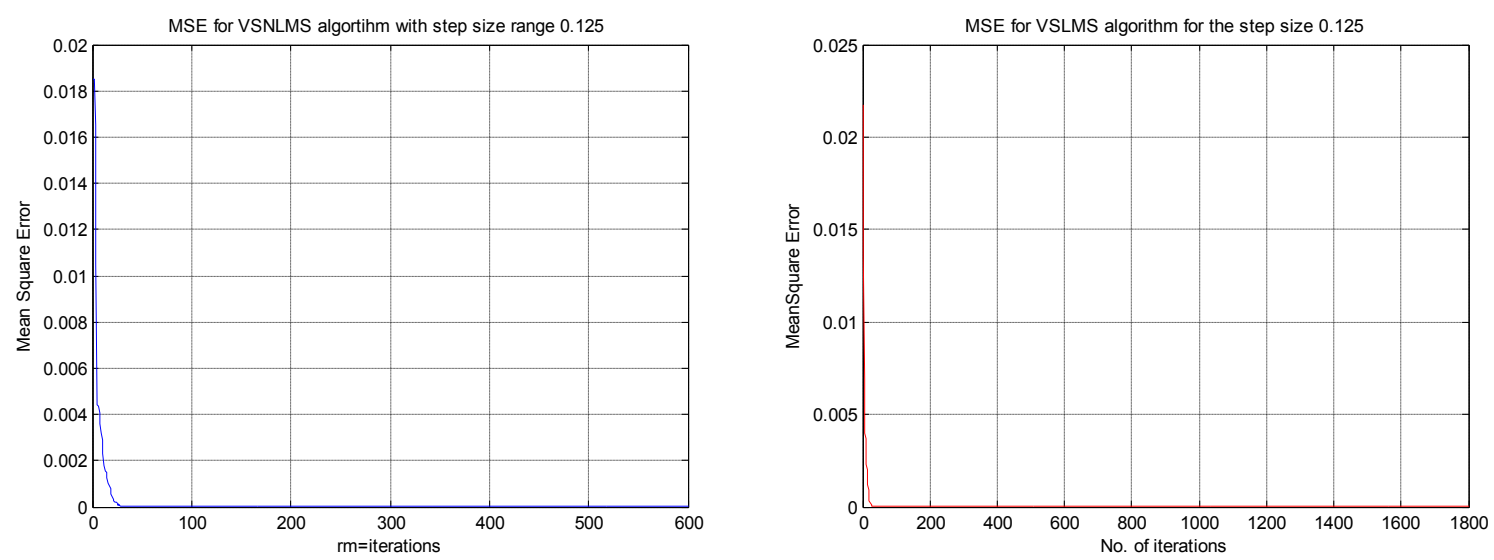

Table1. MSE of Various algorithms for number of iterations $=600,1200$ and 1800.

\begin{tabular}{|c|c|c|c|c|}
\hline Iteration & Applied Algorithm & Step Size & Mean error & $\begin{array}{l}\text { Avg ERLE } \\
\text { (dB) }\end{array}$ \\
\hline 600 & LMS & 0.050 & 0.05163 & -12.8710 \\
\hline 600 & LMS & 0.125 & 0.03505 & -14.5531 \\
\hline 600 & LMS & 0.500 & 0.09041 & -10.4378 \\
\hline 1200 & LMS & 0.050 & 0.04132 & -13.8384 \\
\hline 1200 & LMS & 0.125 & 0.05299 & -12.7581 \\
\hline 1200 & LMS & 0.500 & 0.08390 & -10.7624 \\
\hline 1800 & LMS & 0.050 & 0.03000 & -15.2288 \\
\hline 1800 & LMS & 0.125 & 0.04297 & -13.6683 \\
\hline 1800 & LMS & 0.500 & 0.07039 & -11.5249 \\
\hline 600 & Normalized-LMS & 0.050 & 0.01177 & -19.2922 \\
\hline 600 & Normalized-LMS & 0.125 & 0.04031 & -13.9459 \\
\hline 600 & Normalized-LMS & 0.500 & 0.05047 & -12.9697 \\
\hline 1200 & Normalized-LMS & 0.050 & 0.01780 & -17.4958 \\
\hline 1200 & Normalized-LMS & 0.125 & 0.02354 & -16.2819 \\
\hline 1200 & Normalized-LMS & 0.500 & 0.02983 & -15.2535 \\
\hline 1800 & Normalized-LMS & 0.050 & 0.06708 & -11.7341 \\
\hline 1800 & Normalized-LMS & 0.125 & 0.02847 & -15.4561 \\
\hline 1800 & Normalized-LMS & 0.500 & 0.02574 & -15.8939 \\
\hline 600 & Variable step size-LMS & 0.050 & 0.02059 & -16.8634 \\
\hline 600 & Variable step size-LMS & 0.125 & 0.01673 & -17.7650 \\
\hline 600 & Variable step size-LMS & 0.500 & 0.02059 & -16.8634 \\
\hline 1200 & Variable step size-LMS & 0.050 & 0.01630 & -17.8781 \\
\hline 1200 & Variable step size-LMS & 0.125 & 0.02016 & -16.9551 \\
\hline 1200 & Variable step size-LMS & 0.500 & 0.02039 & -16.9058 \\
\hline 1800 & Variable step size-LMS & 0.050 & 0.03980 & -14.0012 \\
\hline 1800 & Variable step size-LMS & 0.125 & 0.03672 & -14.3510 \\
\hline 1800 & Variable step size-LMS & 0.500 & 0.02120 & -16.7366 \\
\hline 600 & VS Normalized-LMS & 0.050 & 0.00168 & -27.7547 \\
\hline 600 & VS Normalized-LMS & 0.125 & 0.00212 & -26.7469 \\
\hline 600 & VS Normalized-LMS & 0.500 & 0.02246 & -16.4859 \\
\hline 1200 & VS Normalized-LMS & 0.050 & 0.01277 & -18.9381 \\
\hline 1200 & VS Normalized-LMS & 0.125 & 0.01537 & -18.1333 \\
\hline 1200 & VS Normalized-LMS & 0.500 & 0.01893 & -17.2285 \\
\hline 1800 & VS Normalized-LMS & 0.050 & 0.00281 & -25.5098 \\
\hline 1800 & VS Normalized-LMS & 0.125 & 0.02989 & -15.2447 \\
\hline 1800 & VS Normalized-LMS & 0.500 & 0.03098 & -15.0892 \\
\hline
\end{tabular}




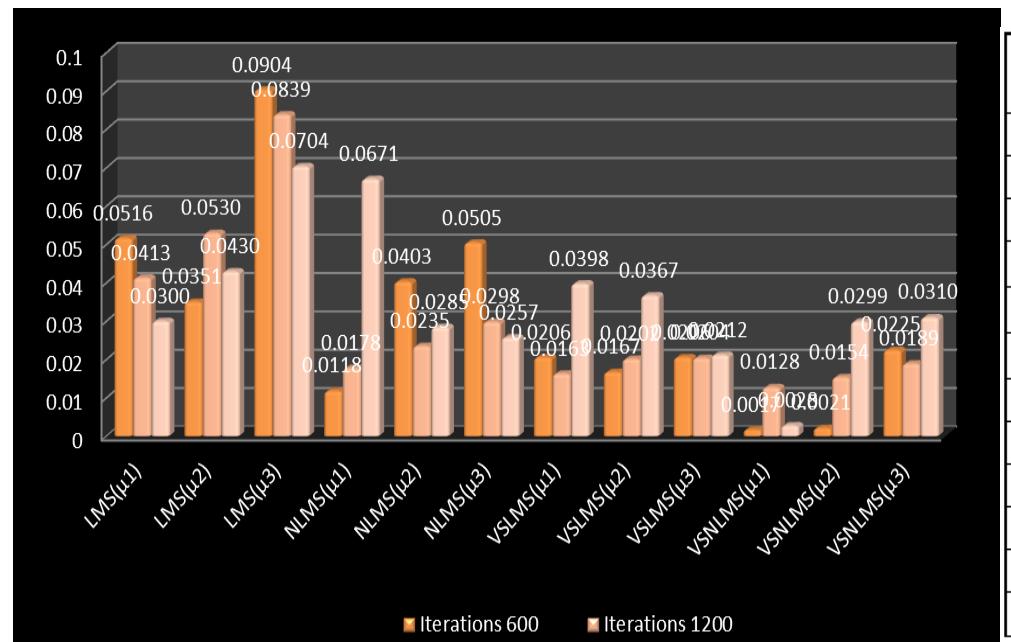

\begin{tabular}{|l|l|l|l|}
\hline $\begin{array}{l}\text { Applied } \\
\text { Algorithm }\end{array}$ & $\begin{array}{l}\text { Iterations } \\
600\end{array}$ & $\begin{array}{l}\text { Iterations } \\
1200\end{array}$ & $\begin{array}{l}\text { Iterations } \\
1800\end{array}$ \\
\hline $\operatorname{LMS}(\mu 1)$ & 0.0516 & 0.0413 & 0.0300 \\
\hline $\operatorname{LMS}(\mu 2)$ & 0.0351 & 0.0530 & 0.0430 \\
\hline $\operatorname{LMS}(\mu 3)$ & 0.0904 & 0.0839 & 0.0704 \\
\hline $\operatorname{NLMS}(\mu 1)$ & 0.0118 & 0.0178 & 0.0671 \\
\hline $\operatorname{NLMS}(\mu 2)$ & 0.0403 & 0.0235 & 0.0285 \\
\hline $\operatorname{NLMS}(\mu 3)$ & 0.0505 & 0.0298 & 0.0257 \\
\hline $\operatorname{VSLMS}(\mu 1)$ & 0.0206 & 0.0163 & 0.0398 \\
\hline $\operatorname{VSLMS}(\mu 2)$ & 0.0167 & 0.0202 & 0.0367 \\
\hline $\operatorname{VSLMS}(\mu 3)$ & 0.0206 & 0.0204 & 0.0212 \\
\hline $\operatorname{VSNLMS}(\mu 1)$ & 0.0017 & 0.0128 & 0.0028 \\
\hline $\operatorname{VSNLMS}(\mu 2)$ & 0.0021 & 0.0154 & 0.0299 \\
\hline $\operatorname{VSNLMS}(\mu 3)$ & 0.0225 & 0.0189 & 0.0310 \\
\hline
\end{tabular}

Figure 3. Graphical analysis of variations in mean error for algorithms for number of iterations=600, 1200 and 1800.

\section{COMPUTATION AND COMPLEXITY}

Application of RBF neural network criteria to eliminate non efficient neuron where neuron is the different algorithm, for the selection of proficient algorithm can be selected for ANC. The performance of the algorithm depends upon the type of input. We found VSNLMS is doing better corresponding to the input 'sound'. Here we used and apply the criteria for deciding threshold value [1]. And the simplified formula is given as

$$
\varphi_{k}(x)=\exp \left(-\left(\frac{1}{\sigma^{2}}\right)\left\|x-\mu_{k}\right\|^{2}\right)
$$

where $\mu \mathrm{k}(\mathrm{x})$ is response of $\mathrm{k}^{\text {th }}$ hidden unit. In our case, implementation of the concept of RBF neural network approach can be used after simplification $\sigma=1$, and mean square error $\mu$ variance is normalized by factor $1 / 20$ Threshold criteria results in selection of the efficiently working neuron as efficient algorithm can be given in simplified manner, where Th is the threshold value and MSE is Mean square error 1/20 is the normalizing factor as :

$$
T h=M S E-\left(\frac{1}{20}\right) \text { average mean }
$$

\section{CONCLUSION AND FUTURE WORK}

It was found out those in particular noisy conditions most suitable algorithm can be sort out using decisive method using Radial basis function in ANC method. This method can also be applied to noise cancellation, vibration cancellation and signal estimation problems. Average of the MSE obtained 0.3175 deviations from mean when calculated; it is observed that Variable step size Normalized LMS is performing better than the other utilized application for ANC of "Sound" input for no. of iterations 1800 with step size 0.5 as the threshold criteria results $=$ Mean error-(1/20)Average. 0.0068 is the least value amongst the all applied algorithm, hence pruning of all other algorithm inculcate the selection of that algorithm as survival neuron. Next good performing algorithm is NLMS itself for 600 iterations of step size 0.05 . The surprisingly good performance of the LMS is due to linear nature of the 'sound' input noise. Here in this project have tried to reduce such weed signals by using basic adaptive approach. But in real time applications which involve thousands of such weed signals and knowing the fact that noise, at any cost, cannot be cancelled to cent percent, this basic approach cannot do us a good favour and keeps this research topic active in upcoming years. The future task is to apply this novel concept for Kalman and Wiener filter's family algorithm simultaneously. It is also being tried to implement it for outer noise removal in automobiles and noise cancellation in head phones. Noise performance can be upgraded by finding proper step factor and threshold value. The algorithm level up gradation of threshold and decisive criteria may improve performance

\section{ACKNOWLEDGEMENTS}

We pay our special thanks to Mr. Himanshu Purohit, Prof. D. Das, for sharing good research ideas and suggestions. We wish to put on record the appreciative original work of all the authors of various technical papers which we have referred in initiation of work without whom it was very difficult to achieve successful completion. 


\section{REFERENCES}

[1] S.M. Kuo and D.R.Morgan, Active Noise Control -Algorithms and DSP Implementations. New York: Wiley, 1996.

[2] Lu Yingwei, N.Sundararajan, P.Saratchandran "Adaptive Nonlinear Systems Identification Using Minimal Radial Basis Function Neural Networks “in IEEE 1996.

[3] Shoji Makino, Member, IEEE, Yutaka Kaneda, Member, IEEE and Nobuo Koizumi, "Exponentially weighted step size NLMS adaptive filter based on the statistics of a room impulse response", IEEE Trans. on speech and audio Processing, vol. 1, No.1, pp.101-108, Jan 1993.

[4] S. Qureshi, "Adaptive Equalization,” Proceedings of the IEEE, vol.73, No.9, pp.1349-1387, Sept. 1985.

[5] S. Haykins, “Adaptive Filter Theory”, Prentice Hall, New Jersey, 1996.

[6] Raymond H. Kwong and Edward W. Johnston, “A Variable Step Size LMS Algorithm”, IEEE Transactions on Signal Processing vol. 40, No.7, pp. 1633-1642, July, 1992.

[7] Tyseer Aboulnasr, Member, IEEE, and K.Mayyas, "A robust variable step size LMS type algorithm: Analysis and Simulations", IEEE Trans. On Signal Processing vol. 45, No.3, pp. 631-639, March 1997.

[8] Dargar S K, Himanshu Purohit, Mahajan "An Iterative Pruning Approach of Neural Network for Proficient Noise Cancellation" in International Journal Engg Advanced Technology, ISSN: 2249 - 8958, Volume-2, Issue-4, April 2013.

[9] Debi Prasad Das ,'Filtered -s LMS Algorithm for Multichannel Active control Of Nonlinear Noise Processes" in IEEE Transactions on Speech And Audio Processing, vol 14 , no.5 September 2006.

[10] Debi Das and Ganpati Panda, "Active Mitigation of Nonlinear Noise Processes Using A Novel Filtered-s LMS Algorithm" in IEEE Transactions of Speech and Audio Processing, vol. 12, No.3, May 2004.

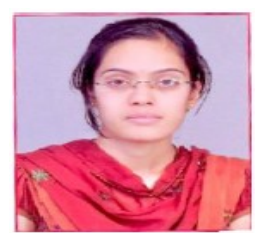

Abha Nyati ${ }^{1}$, is M. Tech scholar from Pacific University PAHER, Udaipur pursuing specialization in Digital Communication. She has completed her degree of Bachelor of Engineering in electronics and communication with honors from Mohanlal Sukhadia University, Rajasthan. She has good academic records and has academic experience in teaching. Her area of research interest is digital communication, signal processing and wireless communication.

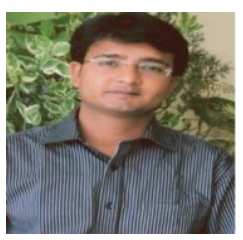

Shashi Kant Dargar ${ }^{2}$, is working as assistant professor in the department of Electronics and Communication Engineering at Sir Padampat Singhania University, Udaipur. Presently, and pursuing Ph.D. He has completed his degree of Bachelor of Engineering from University of Rajasthan, Jaipur in specialization with Electronics and Communication Engineering. He has completed his degree of Masters of Technology in Digital Communication from Rajasthan Technical University, Kota. He has been contributing in academics from seven years. His Area of research interest is signal processing, wireless communication, Microwave \& Communication Engineering.

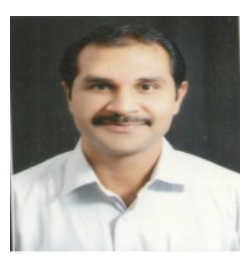

**Deepak Vyas, is working as assistant professor in the department of Electronics and Communication Engineering at Pacific Institute of Technology, Udaipur. Presently, he is pursuing Ph.D. He has completed his degree of Bachelor of Engineering in specialization with Electronics and Communication Engineering. He has completed his degree of Masters of Technology in Digital Communication from Rajasthan Technical University, Kota. He has been contributing in academics form over seven years. His Area of research interest is Communication Engineering. He has guided many projects for undergraduate and post graduate students in his academic career. 